\title{
Tipología de cúpulas tabicadas. Geometría y construcción en la Valencia del siglo XVIII
}

\section{Tile domes typology. Geometry and construction in Valencia 18th century}

\author{
R. Soler-Verdú ${ }^{(*)}$, A. Soler-Estrela ${ }^{(*)}$
}

\section{RESUMEN}

En el contexto de las bóvedas tabicadas, destacan las cúpulas por su protagonismo arquitectónico y particularidades constructivas. La propuesta de tipos constructivos, se ha realizado a partir de una serie de cúpulas tabicadas en las que se han llevado a cabo obras de restauración, lo que ha hecho posible conocer su composición constructiva y geometría. Los resultados obtenidos permiten encuadrarlas como cáscaras extremadamente delgadas, con espesores que alcanzan límites difícilmente superables. Son representativas de centenares de cúpulas levantadas en el siglo XVIII, que tienen como epicentro difusor a la ciudad de Valencia, de acreditada experiencia en la construcción de sistemas de abovedamientos tabicados, desde finales del trescientos. No obstante, las características comunes, hacen que el estudio sea de aplicación en un amplio ámbito hispánico y en un dilatado periodo temporal desde su inicio en el siglo XVI hasta las últimas realizaciones contemporáneas.

Palabras clave: Cúpula; bóveda tabicada; cáscara delgada; albañilería; patrimonio arquitectónico.

\section{ABSTRACT}

In the context of tile vaults, domes are distinguished by its architectural importance and special features. The proposed constructive typology has been made from a series of tile domes where restoration works have been carried out. This fact has made it possible to define its construction detail and geometry. The results obtained situate them as extremely thin shells, with thickness close to the limits. These are representative of hundreds of domes built in the eighteenth century, focused in the city of Valencia, where experience in building tile vaults is proven since the end of the fourteenth century. However common features make the study applicable over the Hispanic area and in a wide period since the beginnings, in the sixteenth century, to the latest contemporary achievements.

Keywords: Dome; tile vault; thin shells; brick masonry; architectural heritage.

(*) Universidad Politécnica de Valencia, (España).

(**) Universitat Jaume I, Castellón de la Plana (España).

Persona de contacto/Corresponding author: rsoler@csa.upv.es (R. Soler-Verdú)

Cómo citar este artículo/Citation: Soler-Verdú, R., Soler-Estrela, A. (2015). Tipología de cúpulas tabicadas. Geometría y construcción en la Valencia del siglo XVIII. Informes de la Construcción, 67(538): eo78, doi: http://dx.doi.org/10.3989/ic.13.180.

Licencia/License: Salvo indicación contraria, todos los contenidos de la edición electrónica de Informes de la Construcción se distribuyen bajo una licencia de uso y distribución Creative Commons Reconocimiento no Comercial 3.o. España (cc-by-nc). 


\section{EL HILO HISTÓRICO}

Un interesante capítulo de las bóvedas tabicadas, es el brillante episodio protagonizado por las excepcionales cúpulas de grandes luces, construidas por Luis Moya (1) que coronan iglesias y edificios públicos. Es un periodo que se inicia en la década de los años cuarenta y se prolonga hasta los sesenta del pasado siglo. Un cierto paralelismo se encuentra en las construcciones tabicadas de Eduardo Sacriste y sus experiencias con la cerámica armada en un intento de conciliar la tradición constructiva y los avances tecnológicos (2).

Luis Moya, apoyándose en la construcción tradicional y reinterpretando soluciones arquitectónicas hispano-musulmanas, introduce innovaciones tecnológicas e investiga nuevas formas, llegando a competir ventajosamente con las estructuras laminares de vanguardia. Moya es un gran conocedor de la historia de los sistemas abovedados, como se comprueba analizando sus cuadernos y su temprana publicación en 1947 sobre Bóvedas tabicadas. Nos interesa destacar que conoció la obra de Guastavino acompañado por el profesor Collins en sus visitas a Estados Unidos y que le debió dejar impronta (3).

Rafael Guastavino ejercía su actividad profesional a finales del siglo XIX, cuando Barcelona era un importante núcleo, en el que destacados arquitectos estaban empleando las bóvedas tabicadas en la construcción de todo tipo de edificios, cuando decidió emigrar a los Estados Unidos. Desde 1885 fueron levantadas con la decisiva contribución de los arquitectos Rafael Guastavino y su hijo, centenares de importantes construcciones cubiertas con bóvedas tabicadas. En colaboración con destacados arquitectos americanos construyeron las cúpulas de edificios tan emblemáticos como la Biblioteca Pública de Boston, la Saint Chapel en la Universidad de Columbia, US Army War College de Washington DC, o la Catedral Saint John the Divine de Nueva York, entre otros muchos (4). Si analizamos su publicación sobre bóvedas tabicadas (5) en el capítulo dedicado a la historia, dedica a la cúpula de Santa María del Fiore de Florencia una sección general y dos dibujos de detalle de los nervios, a la cúpula de San Pedro de Roma una sección alzado general y a la cúpula de San Pablo de Londres una sección sin la linterna. En el texto amplía la relación de cúpulas a las más destacadas añadiendo la Madonna de la Salute de Venezia, Santa Genoveva de Paris y lo que es significativo, incluye junto a tan emblemáticas cúpulas a la Real Capilla de los Desamparados y a la cúpula de los Escolapios ambas de Valencia. A estas dos últimas dedica sendos dibujos. A los Desamparados, planta elíptica y sección y a la Escuelas Pías, planta y sección indicando «cúpula de ladrillo con zuncho de hierro». Esto demuestra que Guastavino admiraba y conocía perfectamente las cúpulas que había contemplado en su juventud, incluso que habría visitado sus desvanes y que conocía la praxis de la albañilería local y que con toda seguridad leía el manual de Fornés (6), que recogía fielmente la tradición centenaria y detallaba el proceso de ejecución de las cúpulas. La bóveda tabicada exportada por los Guastavino reproduce, unas técnicas, oficios y sistemas constructivos inspirados en soluciones de la cultura arquitectónica de siglos precedentes de su Valencia natal, también muy difundidas en ámbito hispano.

Siguiendo el hilo de la historia hay que retroceder hasta el siglo XVI, fecha en la que se inicia la construcción de centenares de cúpulas que tienen a Valencia como importante foco difusor (Figura 1). Para su construcción no se emplea la

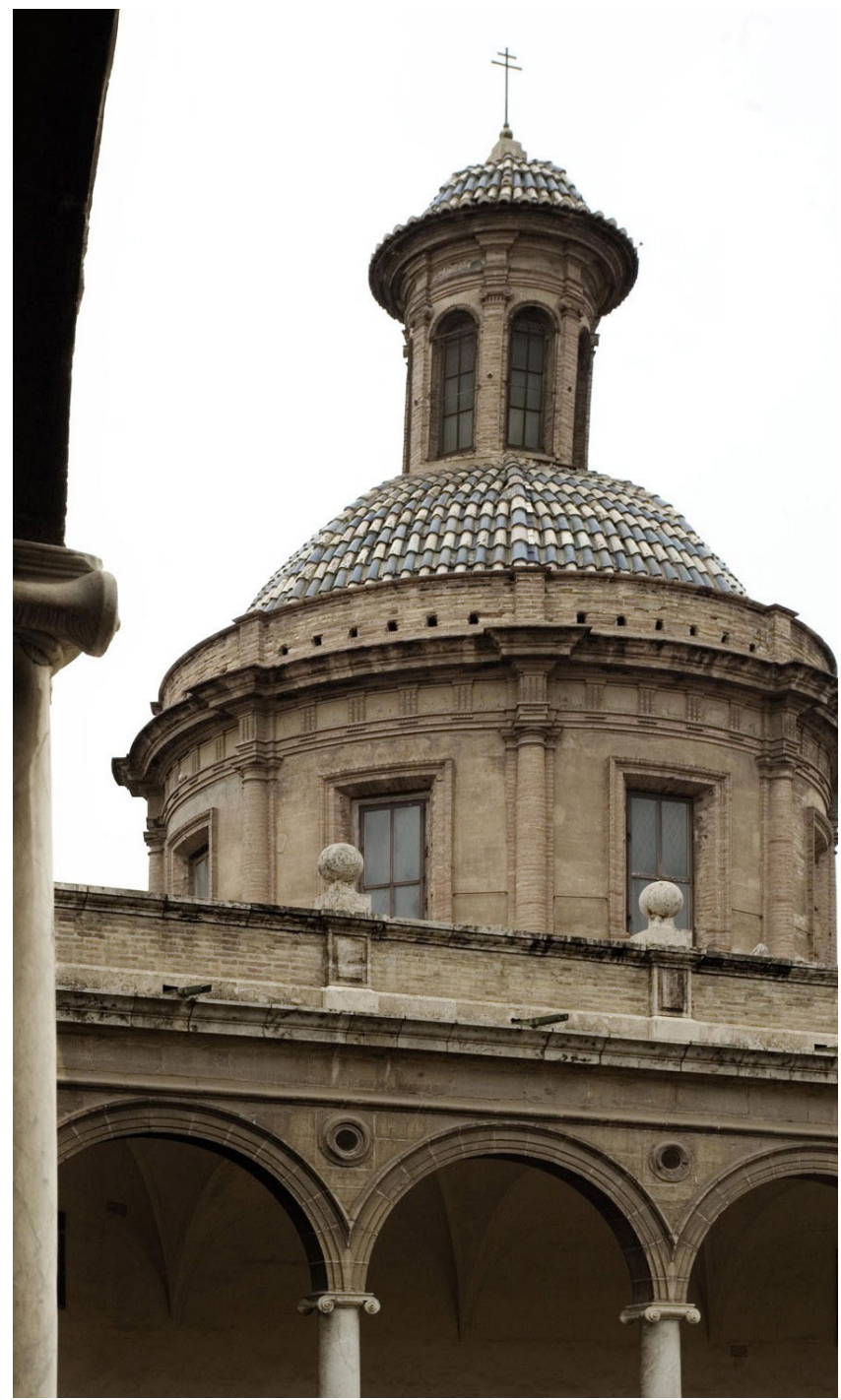

Figura 1. Cúpula del Corpus Christie. Valencia. Finales del siglo XVI.

cantería, sino que se voltean mediante la técnica de la albañilería, y aunque son habituales las construidas con ladrillo a rosca, también son muy frecuentes las ejecutadas mediante bóvedas tabicadas. En contraste con la fama internacional del episodio americano protagonizado por Guastavino, la audacia de estas construcciones y sus alardes técnicos, no han sido suficientemente valorados.

Remontándonos a los orígenes, la primera referencia documental conocida hasta el momento, se refiere a una capitulación para la construcción de una capilla mediante bóveda tabicada en 1382, en el claustro del convento de Santo Domingo de Valencia (7). Esta técnica estaría fuertemente implantada en la Corona de Aragón a lo largo del siglo XIV y el XV, como lo corrobora su empleo frecuente en las edificaciones de dicho período (8). A principios del XVII Fray Lorenzo de San Nicolás recoge en su tratado, con bastante extensión y detalle las bóvedas tabicadas, lo que confirma la generalización de su empleo.

Compartimos plenamente la opinión de José Luis González Moreno-Navarro de que la bóveda tabicada se encuentra en un momento de su historia crítico y su conservación depende de que se comprenda y conozca su técnica constructiva y su comportamiento estructural (9). 
En la actualidad esta centenaria técnica parece de nuevo renacer impulsada por los criterios de sostenibilidad, bajo consumo energético y utilización de recursos locales. Un ejemplo paradigmático es el Mapungubwe National Park Interpretative Centre, del arquitecto surafricano Peter Rich. Un conjunto de edificaciones de grandes cúpulas tabicadas construidas con mano de obra local, que ha merecido el reconocimiento del World Building of the Year from the World Architecture Festival 2010 (10). Las bóvedas tabicadas todavía no han dicho adiós.

\section{METODOLOGÍA}

Los centenares de cúpulas valencianas del setecientos, constituyen un episodio destacable dentro de la historia de la arquitectura y la construcción. Las dificultades de acceso a las cúpulas, tanto desde el interior como desde el exterior, hace que la mayoría de veces no sea posible definir con certeza su composición constructiva, ni tan siquiera su geometría. Definir con precisión los aspectos formales y constructivos sólo es posible en contadas ocasiones y circunstancias.

Las fuentes documentales que hacen referencia a las cúpulas históricas, cuando se trata de fuentes gráficas, relativamente abundantes desde la aparición de la Real Academia de Bellas Artes de San Carlos, son generalmente diseños que no especifican con detalle los aspectos constructivos y prácticamente nunca los procesos de ejecución o los medios auxiliares (11). Los diseños iniciales generalmente sufren importantes modificaciones durante la ejecución, como se tiene constancia comparando las capitulaciones o contratos con la obra finalmente llevada a cabo. La gran envergadura de estas empresas, el dilatado período de tiempo desde el inicio hasta la conclusión o las fuertes limitaciones económicas son factores que contribuyen a ello. Sin olvidar que la construcción es una actividad pragmática condicionada por múltiples factores y limitaciones y donde los oficios participan adaptando o modificando proyectos teóricos durante el proceso de ejecución. Es por lo tanto imprescindible confrontar los diseños iniciales, con el artefacto realmente construido, materializado dentro de un repertorio de soluciones caracterizado por grandes alardes de ligereza, audacia y economía de medios.

La información para elaborar la propuesta de tipología, se ha obtenido por la participación directa de los autores de este artículo en estudios, proyectos de restauración, informes o asesoramientos. Ello ha permitido en las cúpulas seleccionadas la observación directa y en la mayoría de casos al disponer de andamios se ha podido acceder a los desvanes y realizar catas. De esta forma se ha definido la geometría, espesores de las capas, materiales, trasdosados y con precisión los puntos singulares del artefacto realmente construido (12).

\section{TIPOLOGÍA CONSTRUCTIVA}

La cúpula es una cubierta, la envolvente superior de un edificio con la misión de proteger el interior del mismo de las acciones metereológicas. Se trata de un sistema constructivo sometido a exigencias específicas y múltiples, que superan el dominio estrictamente estructural. Desde el punto de vista funcional está sometida a requisitos como estabilidad y resistencia, barrera a la intemperie, comportamiento higrotérmico, barrera acústica, resistencia al fuego, durabilidad o relacionados con el ámbito interior como la iluminación y los acabados decorativos.
Las cúpulas tabicadas construidas, sometidas a las exigencias funcionales enunciadas, responden a un variado repertorio, que hemos clasificado ordenadamente en una propuesta tipológica, en que cada tipo esta contrastado con al menos un caso real perfectamente documentado. La propuesta considera a la hoja como elemento constructivo que configura, con sus diversas conexiones y formas, diversos artefactos clasificables en tipos.

Definimos como hoja al elemento constructivo formado por diferentes capas yuxtapuestas de ladrillo y de pasta de yeso, limitadas por el ámbito interior, el exterior o desvanes. No emplearemos la palabra hoja para definir una capa de ladrillo.

Antes de describir los distintos tipos desarrollaremos los rasgos comunes a todos ellos, como son los materiales empleados y el elemento constructivo base

\subsection{El elemento constructivo. La doble capa}

El análisis de los casos estudiados permite comprobar que la disposición constructiva utilizada casi sin excepción para la formación de las hojas tabicadas es de doble capa de ladrillo de pequeño espesor o rasilla (Figura 2). En la primera, la rasilla se dispone con la junta continua en la dirección de avance tomada con pasta de yeso en su canto y testa (13), un trasdosado de capa fina de pasta fluida de yeso y otra capa de ladrillo a rompejuntas con la primera, realizada casi simultáneamente para asegurar la estabilidad de la bóveda en fase de ejecución. Es precisamente esta disposición la que resuelve la debilidad de la junta de una sola capa, obteniéndose un elemento resistente en el que las capas están unidas gracias a la gran adherencia de los materiales y la técnica empleada durante su ejecución. La segunda capa de ladrillo recibe un llafardat, un recubrimiento superior de pasta de yeso extendido con la mano y por el interior en fase de acabados, un tendido de yeso que evita la caída de alguna rasilla que haya perdido adherencia además de mejorar el comportamiento acústico, resistencia al fuego y convertirse en el soporte de motivos decorativos de pinturas, fingidos y dorados.

Esta disposición de dos capas de rasilla corresponde además a la primera cita documental conocida de la bóveda tabicada «dos raioles dobles de pla» que recoge su utilización en 1382 en el claustro del convento de Santo Domingo de Valencia (7) y que reiteradamente se recoge en capitulaciones de obras de los siglos posteriores.

El manual de época tan tardía, mediados del XIX, de Manuel Fornés (6) dedica un apartado a «Instrucciones sobre la elaboración de las bóvedas tabicadas» que describen el proceso de ejecución. Resalta el fraguado instantáneo del yeso, la perfecta unión y como el conjunto forma un cuerpo sólido. Recomienda que para evitar la caída de ladrillos durante el proceso de ejecución se realice simultáneamente la segunda capa. También realiza una importante observación «y dejar varios días sin realizar los trasdosados debido a la hinchazón del yeso» (14).

Tanto las catas realizadas como las capitulaciones o contratos de obra reiteran la solución constructiva de la doble capa de ladrillo dispuesto de plano tomado con yeso como el elemento constructivo base, sin que se produzca variación en el periodo temporal que abarca al menos desde el siglo XIV hasta el XIX. Cabe recordar que Antoni Gaudí empleaba la 
bóveda tabicada con singular maestría en sus incomparables construcciones y sobre ella decía: «La bóveda tabicada es el elemento mas precioso de nuestra construcción; permite ejecutar con simplicidad y rapidez las formas más complejas, no exige cimbras y tiene gran resistencia en relación con su ligereza y con la simplicidad de sus componentes» (15).

\subsection{Los materiales}

En los estudios realizados de las muestras obtenidas en las obras de restauración, se concluye que los únicos materiales empleados son el ladrillo y el yeso confirmando las citas de los manuales y capitulaciones de obra. Una de las indudables ventajas de las bóvedas tabicadas es su economía por el empleo de materiales tan abundantes como el ladrillo y el yeso. El empleo del yeso ha sido objeto de críticas, muy comunes en tratados de influencia francesa, acerca de su escasa resistencia, su mal comportamiento frente al agua y por la necesidad de adoptar una serie de precauciones durante el proceso de ejecución. Por eso tiene interés que en su tratado B. Bails afirme que «se labran no con mezcla, si con yeso que fragua y se seca con suma brevedad» (16).

En el ámbito geográfico valenciano, la piedra algez calcinada, es un yeso calizo que tiene una tradicionalmente acreditada calidad. «... particularmente en este país, en que los materiales de ladrillo y yeso llevan suma ventaja a los de otras provincias» (17) aunque convendría recordar que no en todos los lugares la piedra algez tiene la calidad adecuada, ni hay un conocimiento de todo el proceso de elaboración, ni existe la tradición de su empleo por parte de una mano de obra experta. Fray Lorenzo de San Nicolás afirma (18) «tornándolo a recocer, que en el Reyno de Aragón llaman vizcocho; y esto quantas vezes se cuece, tanto es mejor; mas no en todas tierras es una misma conveniencia, porque yo hize la experiencia en Madrid, tierra donde aprendí esta facultad, y no tenia la fuerça de lo demás».

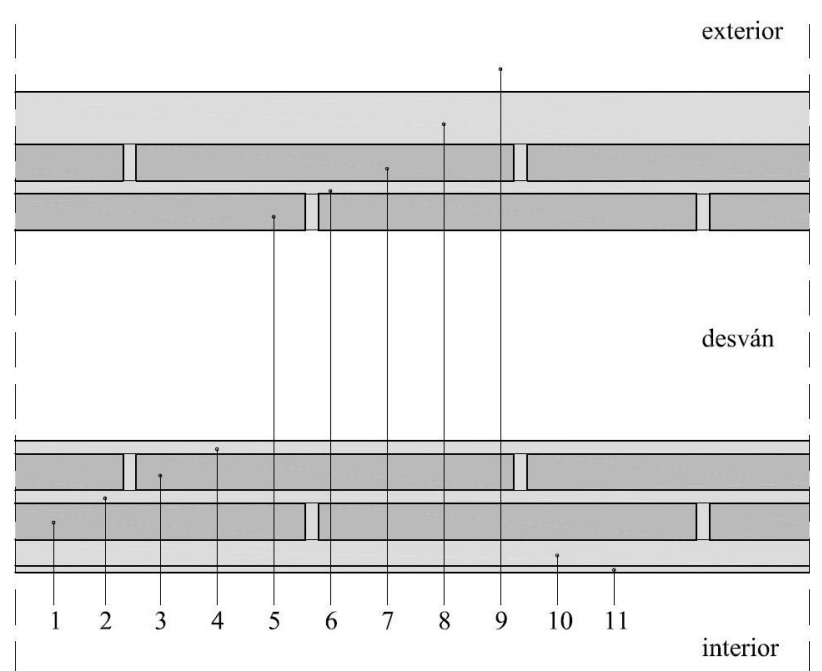

Figura 2. El elemento constructivo.

1: Ladrillo macizo, $29 \times 14 \times 3 \mathrm{~cm}$, tomado con pasta de yeso. 2: Capa fina de yeso, $10 \mathrm{~mm}$.

3:Ladrillo macizo, $29 \times 14 \times 3 \mathrm{~cm}$, tomado con pasta de yeso. 4: Capa irregular de yeso, extendida manualmente.

5: Ladrillo macizo, $29 \times 14 \times 3 \mathrm{~cm}$, tomado con pasta de yeso. 6: Capa fina de yeso, $10 \mathrm{~mm}$.

7: Ladrillo macizo, $29 \times 14 \times 3 \mathrm{~cm}$, tomado con pasta de yeso. 8: Capa de mortero de yeso de $40 \mathrm{~mm}$ 9: Revestimiento de teja.

10: Guarnecido de yeso, $20 \mathrm{~mm}$. 11: Pinturas, dorados o estucos.
Aunque es habitual utilizar el término «ladrillo»y así se viene haciendo en este artículo, sería quizás más apropiada la denominación de rasilla más acorde con el término empleado en la documentación histórica rajola que su primera acepción es la de ladrillo de poco espesor. Más allá de precisiones terminológicas, se está haciendo referencia a una pieza maciza de material cerámico cocido, de fabricación artesanal y con dimensiones que presentan ciertas variaciones incluso en una misma cúpula, pero cuyas dimensiones medias habituales están próximas a $30 \times 14$ y de un espesor menor que $3 \mathrm{~cm}$.

\subsection{Los tipos}

La disposición de las hojas tabicadas y su trasdosado es la variable que permite diferenciar los distintos tipos, que pasamos a describir.

\subsubsection{Tipo A. Trasdosadas}

En estos casos, la cúpula tabicada cierra el espacio interior creando un desván, que queda protegido del exterior por la cubierta que suele responder a una geometría simple, soportada por una estructura independiente del sistema de abovedamiento interior. La calota tabicada deberá de recibir delgadas capas, generalmente guarnecidos, dorados y pinturas. Revestimientos decorativos de gran importancia para la imagen arquitectónica pero desde el punto de vista estructural la calota únicamente debe de soportar los pesos propios, se trata de la hoja interior que cierra inferiormente un espacio cuya envolvente se materializa con otras técnicas (Figura 3).

\section{Tipo A1. Trasdosada por cubierta de carpintería}

Podemos considerar como un precedente de este tipo a los sistemas abovedados de las naves de las catedrales centro europeas, que son en cierto modo un falso techo que cierra inferiormente unos grandes desvanes transitables de unas cubiertas que se resuelven con estructuras de carpintería de armar. En el tipo A1 la cúpula es de una sola hoja interior que cierra el espacio creando un desván y no sobresalen al exterior, sólo ocasionalmente la linterna. Su calota únicamente debe de soportar su propio peso y está protegida del exterior por la cubierta que es un tejado soportado por una estructura de madera independiente del sistema de abovedamiento interior.

Este tipo lo recoge Fray Lorenzo de San Nicolás al enunciar sus sistemas de abovedamientos y le dedica una lámina. Es un tipo que tiene una amplia utilización en ámbito hispano. La cúpula oval del crucero del Hospital General de Valencia (Figura 3, A1), es con toda probabilidad la cúpula de ladrillo tabicado más temprana en el ámbito hispánico, datada de principios del quinientos (19).

La calota tabicada de directriz rebajada, es una hoja interior de doble capa y el tejado exterior es una cubierta descompuesta mediante limas en faldones que evacuan linealmente por la cornisa.

$\mathrm{Al}$ mismo tipo pertenecen las bóvedas situadas en las capillas de los brazos del crucero del Ermitorio de San Marcos, de Olocau del Rey por cuyo desván hemos transitado (Figura 4). 

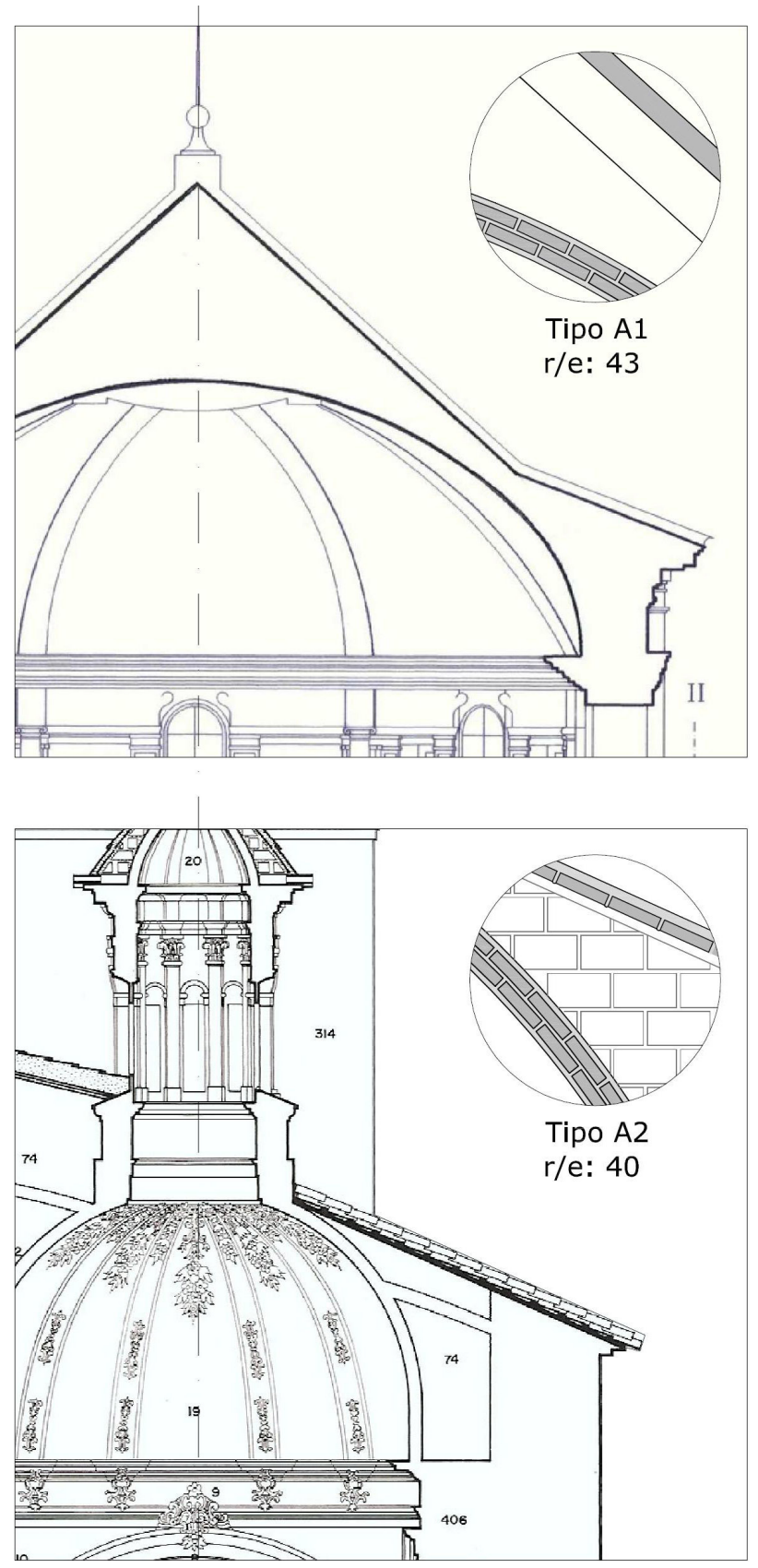

Figura 3. Tipo constructivo: A1, Crucero del Hospital General, Valencia; A2, Iglesia de Santo Tomás y San Felipe Neri. Valencia.

\section{Tipo A2. Trasdosada por cubierta de albañilería}

En este caso, aunque perteneciendo al mismo tipo funcional, el oficio y las técnicas de la albañilería sustituyen a la carpintería de armar, en una actuación pragmática que introduce interesantes matices propios. La cúpula queda trasdosada por tabiquillos, tomando prestada una solución del rico repertorio de los abovedamientos de las naves de las iglesias valencianas frecuentes desde el siglo XVII, y de las que se tenía una acreditada maestría. El artefacto resultante es de un sofisticado comportamiento de la hoja interior tabicada y el tablero superior conectados por un entramado de tabiquillos.

La sustitución de la madera, con una durabilidad afectada por habituales pudriciones y eventuales incendios, por materiales como el ladrillo y el yeso, es una notable mejora que ha

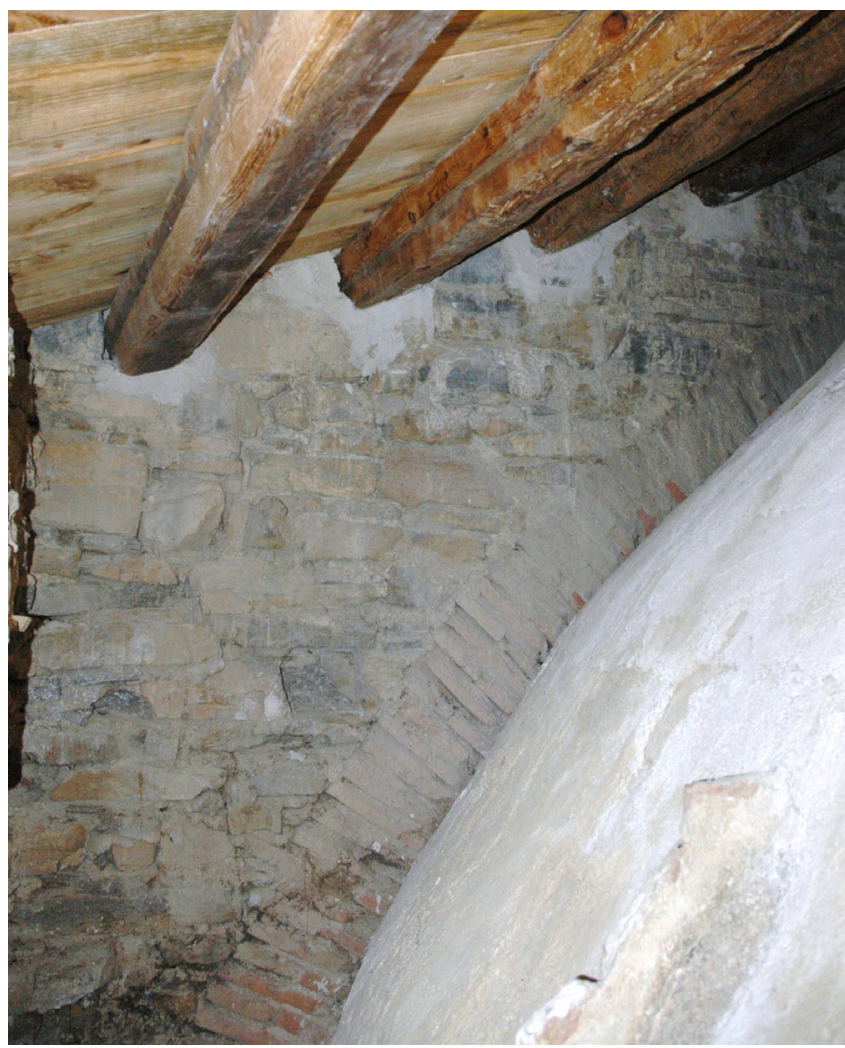

Figura 4. Cúpula capilla trasdosada por entramado de madera. Olocau del Rey, Castellón.

contribuido a su conservación hasta nuestros días, incluso sin apenas mantenimiento.

A este tipo pertenece la Iglesia de Santo Tomás y San Felipe Neri de Valencia, atribuida al ilustrado T. V. Tosca (Figura 3, A2). En las capillas laterales, encajadas entre los contrafuertes de la nave mayor, la cúpula es de una hoja de tabique doble, de forma semiesférica de 21 palmos de diámetro (palmo valenciano 22,65 cm). Los tabiquillos que trasdosan la cúpula, arrancan desde bóvedas tabicadas intermedias apoyadas en los muros perimetral. En este caso sobresale la linterna coronada con cupulín del faldón de la cubierta. El sistema de abovedamiento, yuxtaposición de bóvedas y tabiquillos (Figura 5) es de una relativa complejidad, pero de excelente comportamiento mecánico estructural e higrotérmico (20).

\subsubsection{Tipo B. Sin trasdosar}

La cúpula no solo define el ámbito interior, sino que simultáneamente se manifiesta al exterior y debe servir de soporte al revestimiento de teja. El artefacto constructivo está sometido a múltiples y complejas exigencias funcionales, dada su condición de cubierta, a la vez que forma parte del ámbito interior. La subclasificación que se propone toma a la hoja como elemento constructivo que configura con sus diversas conexiones y formas, distintos artefactos.

Dentro de este tipo hay que distinguir entre cúpulas de una hoja de las de dos, en las que debe diferenciarse la hoja interior de la exterior. La posición de la hoja tiene gran trascendencia desde el punto de vista funcional, de mayor relevancia que las diferencias debidas a sus espesores o capas que las forman (Figura 6). 


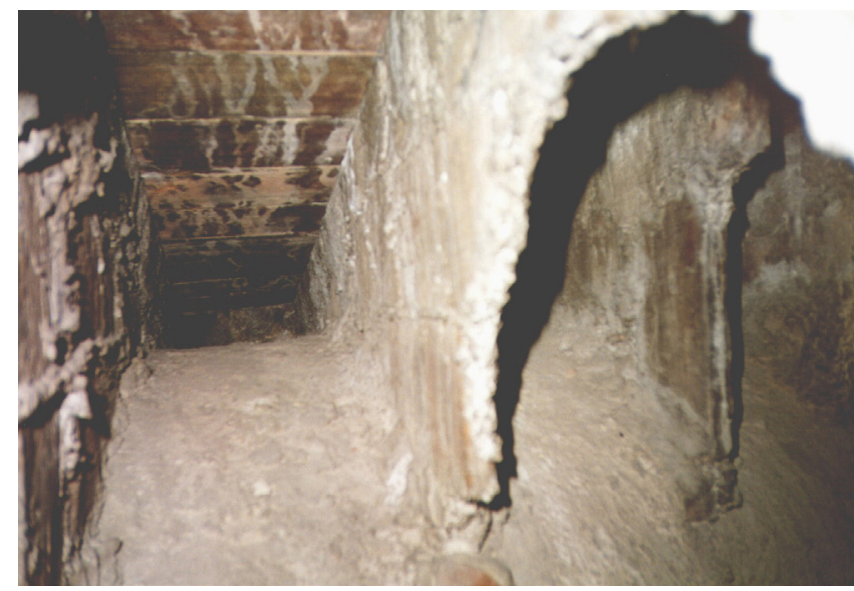

Figura 5. Hoja interior trasdosada por tabiquillos. Iglesia de San Roque, Oliva, Valencia.

\section{Tipo B1. Sin trasdosar. De una hoja simple}

En las cúpulas de una sola hoja, ésta responderá satisfactoriamente a todos los requisitos exigibles a su doble condición estructural y de cubierta. Su intradós incorpora los acabados decorativos y pictóricos de extraordinaria importancia en la imagen interior del templo y simultáneamente su revestimiento exterior, debe de resolver complicados problemas de fijación de la teja y descomposición de superficies no desarrollables en faldones.

A esta clase pertenece la cúpula de la capilla de Comunión de la Iglesia de San Bartolomé, de La Todolella, Castellón (Figura 6, B1). La cúpula se eleva sobre tambor cilíndrico apeado por pechinas y arcos torales. Su diámetro es de 20 palmos valencianos, de directriz semiesférica, que se eleva desde una cornisa interior situada en un plano inferior a la cornisa del tambor. La hoja es de doble capa de ladrillo de $3 \mathrm{~cm}$, que recibe un tendido de yeso por su interior y es trasdosada exteriormente con una capa de mortero de yeso de espesor medio de $3 \mathrm{~cm}$, sobre el que se dispone la teja. El ladrillo está tomado con yeso y ambas capas están unidas mediante una pasta de yeso de mínimo espesor (21).

\section{Tipo B2. Sin trasdosar. De una hoja compuesta}

En ocasiones, para dar mejor respuesta a las múltiples exigencias funcionales y constructivas, aparecen soluciones que utilizan una hoja que se puede considerar compuesta, con la formación de una especie de cámara, al disponer de una pequeña costilla sobre la hoja para apoyo de un tablero de ladrillo. Esta disposición constructiva es reciclada dos siglos después por Eladio Dieste (2). Como una solución tecnológica y funcional eficiente. Cabría una interpretación como calota de dos hojas, pero la disposición constructiva y su íntima conexión constructiva y geométrica implica un comportamiento unitario. Puede entenderse siguiendo una línea evolutiva como una transición entre las cúpulas de una y de dos hojas.

Un caso representativo es la cúpula de la Capilla del Cristo de la Iglesia de San Roque de Oliva. Valencia (Figura 6, B2). La cúpula principal de la capilla de comunión es de planta circular de 30 palmos de diámetro, siendo su flecha de 22 palmos. Su superficie cabe interpretarse como un elipsoide de revolución. A partir de un anillo de medio pie de espesor a una cota aproximada de 3 palmos por encima de la cornisa exterior, se voltea una bóveda tabicada de doble ladrillo macizo tomada con yeso.
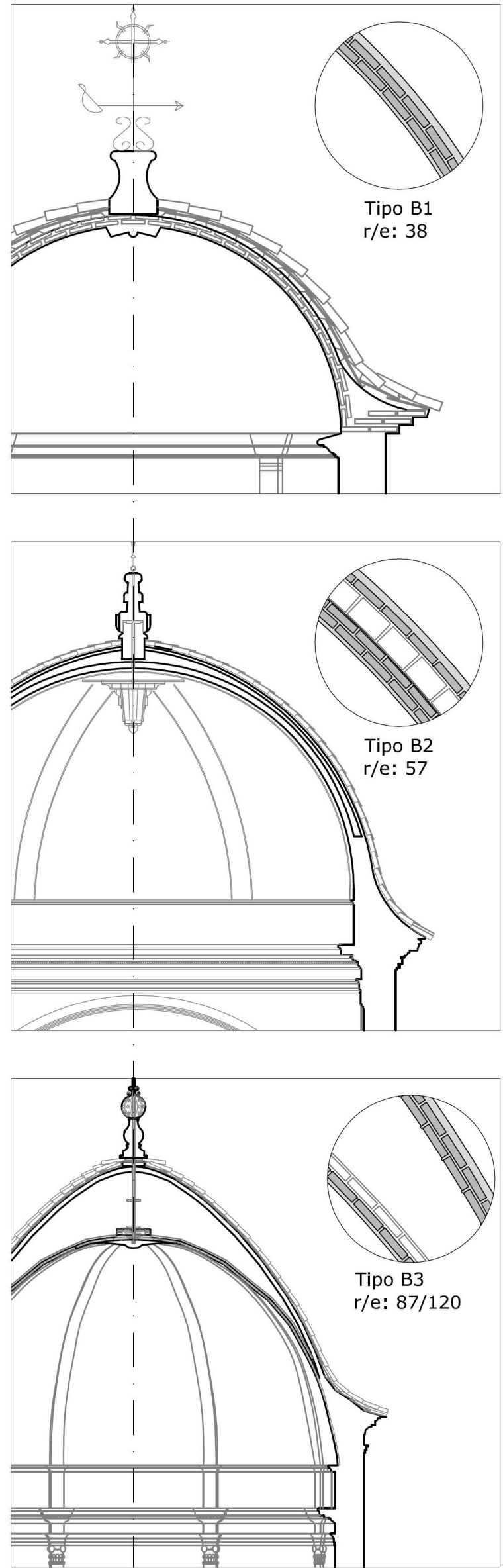

Figura 6. Tipo constructivo B: B1, Iglesia de San Bartolomé en la Todolella, Castellón; B2, Iglesia de San Roque, Oliva, Valencia;

B3, Ermitorio de San Marcos, Olocau del Rey, Castellón. 
Sobre esta doble capa interior, perpendicularmente a cada lado del tambor, se disponen formadas por un panderete de un solo ladrillo unas costillas que sirven de apoyo al tablero superior formado por un ladrillo horizontal tomado y trasdosado con yeso, generando un cajón hueco. Las costillas son perpendiculares a los lados del octógono y convergen en las limas que despiezan en faldones la esfera. El tablero exterior sigue la misma geometría que la doble capa interior sólo separada por el canto de un ladrillo. Es muy sugerente la estructura cajón creada con las costillas, no sólo desde el punto de vista estructural, sino principalmente desde el punto de vista del comportamiento higrotérmico, debido a la formación de la cámara (22).

\section{Tipo B3. Sin trasdosar. De dos hojas}

Este tipo se identifica con facilidad por la existencia de un desván o cámara de aire. Las dos hojas pueden complementarse para resolver aspectos estructurales o de evacuación de las aguas, o los relativos al ámbito interior. El volteo de ambas hojas puede realizarse con algunas conexiones, a nivel de arranque, en la coronación o en las limas y sus superficies pueden generarse con distintas geometrías. Los artefactos de este tipo pueden alcanzar una notable complejidad constructiva para salvar grandes luces con espesores mínimos.

La hoja exterior sirve de soporte al tejado y está expuesta a las acciones meteorológicas, mientras que la hoja interior está protegida por el desván y tiene unas condiciones muy ventajosas respecto a su comportamiento estructural ya que tan sólo debe ser autoportante.

Hemos escogido tres casos que ilustran evoluciones de este tipo.

- Iglesia de la Asunción. Crucero. Carcaixent, Valencia. La cúpula interior, erigida a mediados del siglo XVII, es una semiesfera de 50 palmos de diámetro que arranca a partir de un plano elevado unos tres palmos de la cornisa interior. Está ejecutada con fábrica de ladrillo de pie y medio de espesor. Exteriormente, sin conexión con la cúpula interior, se voltea en fecha desconocida, una bóveda tabicada de doble capa, de hiladas horizontales, de directriz semiesférica de 54 palmos de diámetro, cuyo centro se sitúa elevado 6 palmos respecto de la cornisa interior. En el momento de la toma de datos para el estudio (23), la hoja interior tenía desprendida una capa de ladrillo en una zona junto al arranque de aproximadamente dos metros cuadrados debido a problemas de entrada de agua por diversas grietas en dirección meridiana (Figura 7).

- Ermitorio de San Marcos. Crucero. Olocau del Rey, Castellón (Figura 6, B3). La cúpula central se eleva sobre un esbelto tambor, de planta exterior octogonal. El diseño de la cúpula toma como diámetro 40 palmos valencianos. La hoja interior responde a la geometría de una semiesfera, que arranca con zócalo vertical de tres palmos sobre una imposta interior. La hoja exterior obedece a una geometría peraltada, con una flecha de dos tercios de la luz. La solución constructiva se materializa mediante dos hojas tabicadas. La interior de un solo ladrillo de $3 \mathrm{~cm}$ de espesor revestido con yeso por ambas caras. Presenta nervios decorativos por el interior, mientras que por su extradós recibe un suplemento de ocho arcos de ancho variable, de un doblado de ladrillo revestido con yeso, que confluyen formando una corona superior. La hoja exterior es de dos capas de ladrillo revestida en este caso por el exterior con

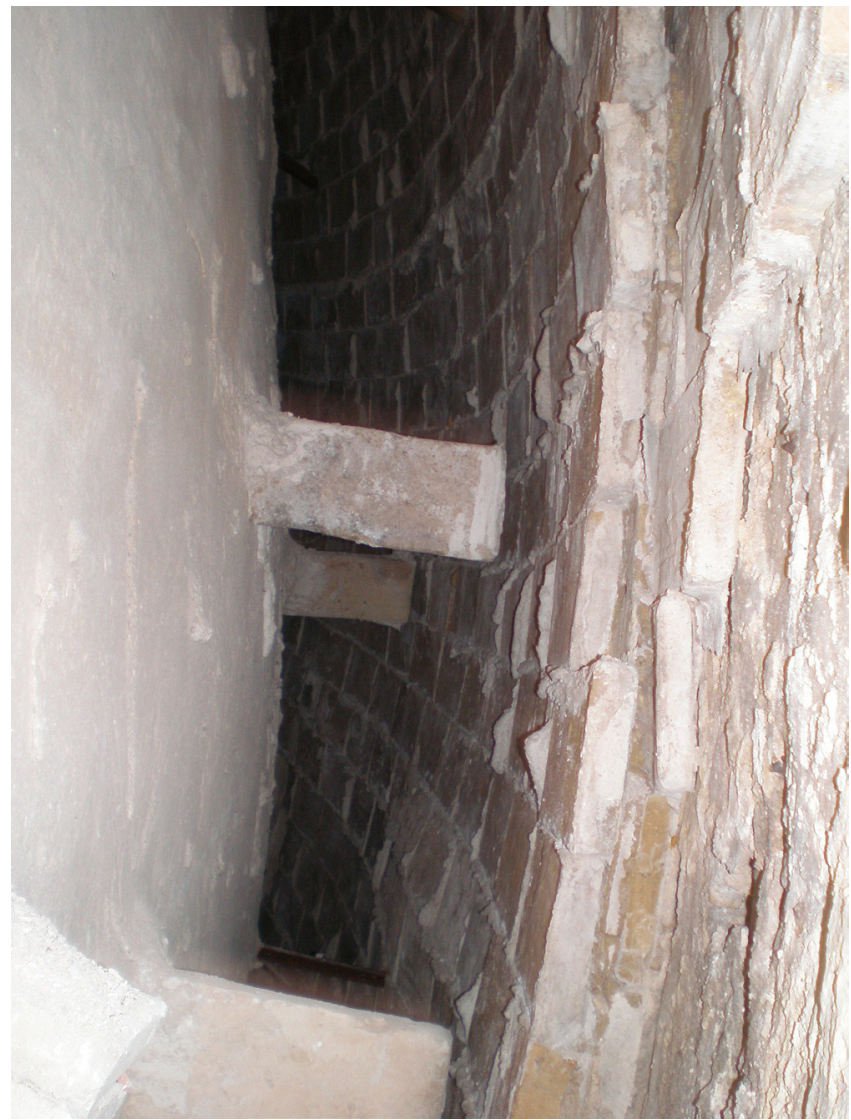

Figura 7. Iglesia Asunción. Carcaixent. Hoja exterior tabicada. Espesor $6 \mathrm{~cm}$, diámetro $12 \mathrm{~m}$.

una capa de mortero de yeso. En la zona de arranque de la cúpula ambas hojas están yuxtapuestas para separarse a la altura aproximada de un metro y medio, generándose un desván entre ellas, sin ninguna conexión entre las hojas a partir de dicha cota (24).

- Iglesia Arciprestal San Jaime. Crucero. Vila-real, Castellón. Sobre los arcos torales y las pechinas del crucero, se eleva un tambor de traza octogonal en su exterior y circular por el interior, cuya cornisa se eleva aproximadamente tres metros y medio sobre el plano de arranque de la cúpula, que tiene como diámetro la medida de 54 palmos valencianos equivalentes a $12 \mathrm{~m}$. La hoja interior es de sección vertical peraltada que genera una superficie de revolución, que es vaciada mediante ocho lunetos, en correspondencia con los ventanales del tambor. El intradós es compartimentado por ocho nervios pareados. La composición de la hoja interior responde a una bóveda tabicada de doble ladrillo, tomado y revestida con yeso. La hoja exterior obedece a una geometría peraltada, alcanzando una altura de $12 \mathrm{~m}$ coincidente con el diámetro de la misma. Está conformada por ocho bóvedas con aristas coincidentes con las diagonales del tambor y en cuya intersección se sitúan ocho costillas, tabiques a panderete, que arrancan desde la hoja interior y convergen en un pilar de ladrillo de planta cuadrada de pie y medio de lado. También es una bóveda tabicada, de ladrillo doble, similar a la descrita en la hoja interior. Presenta la particularidad en la zona inferior desde el arranque, hasta un nivel de 1,60 m superior a la cornisa de la cubierta, de estar trasdosada por unos tabiquillos de ladrillo macizo, formando unos faldones de transición de la planta circular a la octogonal (Figura 8). 

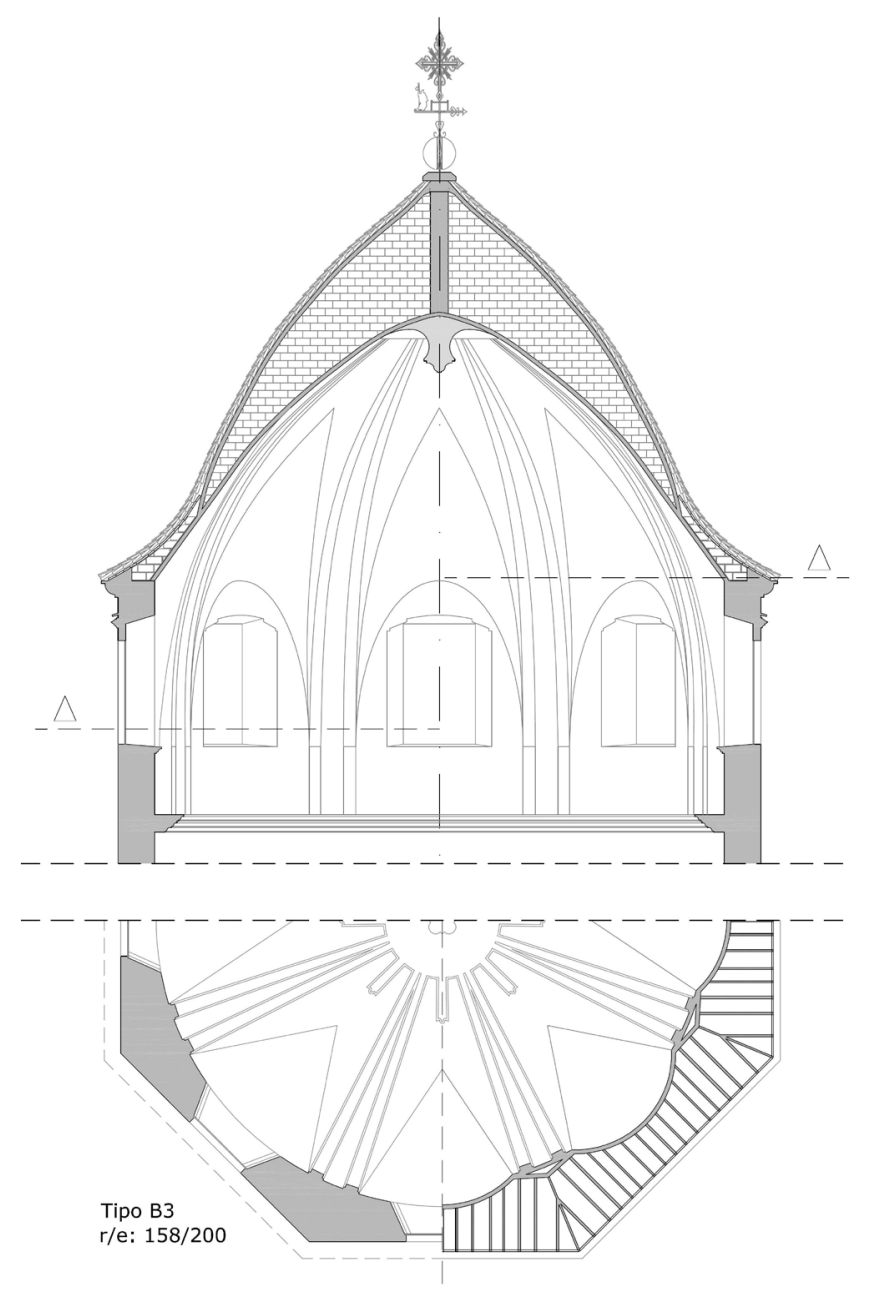

Figura 8. Cúpula Arciprestal Vila-Real. Secciones vertical y horizontal.

El sistema generado por las bóvedas de la hoja interior, los lunetos, la hoja exterior, las costillas de conexión y los tabiquillos exteriores, generan un artefacto de gran complejidad constructiva y de grandes prestaciones a los efectos del comportamiento mecánico estructural y a su funcionamiento higrotérmico (25).

\section{LA CALOTA RESISTENTE}

\subsection{Geometría y dimensiones}

En una cúpula su calota resistente es de extraordinaria importancia, es el soporte estructural. La cúpula es un caso de bóveda de doble curvatura, lo que confiere a su calota una característica esencial a los efectos del comportamiento mecánico, en las tabicadas hay que destacar su pequeño espesor en comparación con las dimensiones de la luz del espacio a cubrir. Los expertos en cálculo de estructuras históricas consideran las cúpulas como cáscaras delgadas, cuando la relación entre el radio y el espesor es mayor a 20, pudiéndose aplicar el análisis según la teoría de membrana (26).

En todas las cúpulas analizadas el espesor más representativo que sirve de referencia es la doble capa de ladrillo, que unidas por la capa de yeso alcanza un espesor de aproximadamente $6 \mathrm{~cm}$. Se podría sumar el tendido inferior que se realiza posteriormente y el trasdosado para anclaje de la teja pero no está muy clara su contribución a la resistencia y en todo caso son inexistentes en la primera y decisiva fase de ejecución en la que únicamente existe la doble capa de ladrillo. Ocasionalmente hay soluciones en las que el espesor es de una sola capa reforzada por nervios planos que constituyen una segunda capa y es excepcional el caso de una sola capa aunque la hemos encontrado en dos cúpulas. En las soluciones de hoja compuesta puede aceptarse desde el punto de vista de la seguridad como de doble capa, lo mismo sucede cuando es trasdosado por tabiquillos, no considerando su indudable colaboración.

Las cúpulas semiesféricas predominan, no obstante aparecen algunas cúpulas peraltadas y rebajadas, pero que responden a una sección vertical de radio conocido, valor que se ha tomado para relacionarlo con el espesor.

Los valores de la relación entre el radio y el espesor de la calota en las cúpulas documentadas en las restauraciones realizadas es de: 38, 40, 43, 57, 87, 120, 158, 200 (Figuras 3, 6 y 8). Todos mayores que el valor establecido para la cáscara delgada de 20. Los resultados de las cúpulas estudiadas permiten encuadrarlas sin ninguna duda como cáscaras extremadamente delgadas, en ocasiones con espesores que alcanzan limites difícilmente superables, teóricamente cero a partir de valores superiores a 50 (27).

Otra cuestión que sólo enunciaremos, es que los estudios estructurales no pueden limitarse a contemplar las acciones gravitatorias. Las hojas exteriores están sometidas a inevitables acciones térmicas. De hecho son las más importantes, como ya reconocía Luis Moya (3).

\subsection{Los puntos singulares}

Para que los estudios de estabilidad, seguridad y resistencia respondan al comportamiento real de la cúpula analizada, hay que detallar la conexión de la calota resistente tabicada con el resto de elementos del sistema constructivo, especialmente respecto al tambor y analizar los puntos singulares.

- El arranque. Se ha tenido una especial atención para detallar el arranque de la cáscara resistente respecto al tambor que la envuelve. El volteo de la cúpula se realiza desde una imposta interior que se sitúa en un plano notablemente inferior al de la cornisa exterior del tambor. Esta disposición constructiva, trasdosa la cúpula en su arranque hasta una cierta altura, por lo que la calota resistente en su banda inferior, hay que considerarla en toda su complejidad incorporando la fábrica del tambor, sin suponerla aislada como en la parte superior. Los modelos de cálculo al establecer las condiciones de contorno deben de reflejar con fidelidad la conexión y modo de apoyo de la cúpula, precisamente en esta banda inferior, que puede ser crítica, ya que si se individualiza la cúpula como si fuera inexistente el tambor, aparecen tracciones que no puede soportar la cáscara resistente que no responden al artefacto construido.

- La coronación. La coronación de la cúpula es un punto singular que suele recibir cargas concentradas. En el exterior se colocan diferentes remates decorativos: pedestales de piedra, cruces, veletas y vástagos metálicos que atraviesan su calota para penetrar en el interior, siendo estabilizados mediante diversos dispositivos. Por su intradós suelen recibir decoraciones de grandes florones o esculturas 
suspendidas de gran peso. Además es una zona de mínima pendiente y con frecuente entrada de agua. Todo lo anteriormente descrito recomienda un conocimiento detallado de las disposiciones constructivas de cada caso entre la calota y los citados elementos, y suelen ser puntos que requieren ser reforzados.

- Los trasdosados. En algunos tipos de cúpulas, la hoja es trasdosada para la formación de los faldones de la cubierta de teja. Esta solución constructiva permite compatibilizar la geometría de la cúpula con la de la cubierta exterior, de planta poligonal. Para ello se dispone de un entramado de tabiquillos conejeros sobre los que se apoyan ladrillos que materializan un tablero que forma las pendientes de los faldones que despiezan la cubierta. El trasdosado transforma la hoja de doble capa, en un nuevo elemento cajón de grandes prestaciones estructurales e higrotérmicas.

\section{CONCLUSIONES}

Los casos considerados son representativos de centenares de cúpulas levantadas en el siglo XVIII, que tienen como epicentro difusor a la ciudad de Valencia. No obstante, las características comunes, hacen que el estudio sea de aplicación en un amplio ámbito hispánico y en un dilatado periodo temporal desde su inicio en el siglo XVI hasta las últimas realizaciones contemporáneas.

La bóveda tabicada formada por tan solo dos capas de ladrillo unidas con yeso, es el elemento constructivo generalizado. Esta disposición se utiliza en cúpulas de muy distintas dimensiones por lo que responde a criterios constructivos más que estructurales. Los resultados obtenidos permiten encuadrarlas como cáscaras extremadamente delgadas, con espesores que alcanzan límites difícilmente superables.

La definición de un tipo es una metodología que va más allá del caso concreto y de la generalización abstracta. Los tipos constructivos estudiados son fuente inagotable de investigaciones propiciando formular, desde la ciencia de la construcción, modelos teóricos que confronten las múltiples exigencias funcionales con el artefacto construido. El análisis de las cúpulas tabicadas solo puede entenderse teniendo en cuenta el detalle constructivo real, y el modo de resolver los puntos singulares de arranque, coronación y trasdosados. La interpretación debe realizarse desde las reglas de la albañilería, no siendo extrapolables las de la cantería con la que, aunque comparte muchos principios, tiene unas diferencias decisivas, desde el proceso de ejecución al comportamiento final.

\section{REFERENCIAS}

(1) García-G, J., Capitel, A. (2000). Luis Moya Blanco. Arquitecto 1904-199o. p. 129, Madrid: Electa.

(2) García, J., González, M., Losada, J.C. (2012). Arquitectura y construcción tabicada en torno a Eduardo Sacriste. Informes de la Construcción, 64(525): 35-50, doi: http://dx.doi.org/10.3989/ic.09.065.

(3) Adell, J. M., Rolando, A. (2005). Luis Moya y las bóvedas tabicadas en la posguerra española. Informes de la construcción, 56(496): 25-29, doi: http://dx.doi.org/10.3989/ic.2005.v57.i496.460.

(4) Ochendorf, J. (2010). Guastavino Vaulting. The Art of Structural Tile. New York: Chronicle Books LLC.

(5) Guastavino, R. (1893). Essay on the theory and history of Cohesive Construction, applied especially to the timbrel vault, Boston: Ticknor and Company.

(6) Fornés, M. (1841). Observaciones sobre la Práctica del Arte de Edificar, p. 46. Valencia: Imprenta de Cabrerizo. http:// www.sedhc.es/biblioteca/tratado.php?ID_pubD=17.

(7) Gómez-Ferrer, M. (2003). Las bóvedas tabicadas en la arquitectura valenciana durante los siglos XIV, XV y XVI. En Mira, E., Zaragoza, A. (Eds.), Una arquitectura gótica mediterránea - Vol. II, (pp. 113-151). Valencia: Generalitat Valenciana.

(8) Zaragoza, A., Ibáñez, J. (2011). Materiales, técnicas y significados en torno a la arquitectura de la Corona de Aragón en tiempos del Compromiso de Caspe (1410-1412). Artigrama, 26: 21-102.

(9) González, J. L. (2005). La bóveda tabicada: entre la conservación y la destrucción. Informes de la construcción, 56(496): 67-72, doi: http://dx.doi.org/10.3989/ic.2005.v57.i496.495.

(10) Ramage, M. H., Oschendorf, J., Rich, P., Bellamy, J. K., Block, P. (2010). Design and construction of the Mapungubwe National Park Interpretative Centre, South Africa. ATDF Journal, 7(1/2):14-23.

(11) Soler, R. (2006). Cúpulas históricas valencianas. En Soler, R. (Ed.), Las cúpulas azules de la comunidad valenciana, (pp. 269-298). Valencia: Generalitat Valenciana.

(12) Soler, R., Soler, A. (2011). Navegando por el trasdós de las cúpulas tabicadas. Tipos constructivos y noticia de artefactos construidos. En Zaragoza, A., Soler, R., Marín, R. (Eds.), Construyendo bóvedas tabicadas, (pp. 176-203). Valencia: Universidad Politécnica.

(13) Truñó, A. (2004). Construcción de bóvedas tabicada, p. 199. Madrid: Juan de Herrera.

(14) Fornés, M. (1841). Observaciones sobre la práctica del arte de edificar, p. 48. Valencia: Imprenta de Cabrerizo. http:// www.sedhc.es/biblioteca/tratado.php?ID_pubD=17.

(15) Bergós, J. (1953). Materiales y elementos de construcción, p. 252. Barcelona: Bosch.

(16) Bails, B. (1796). Elementos de Matemáticas. Tom. IX. Parte I. Que trata de la Arquitectura Civil. 2. ${ }^{\text {a }}$ Ed. corregida, p. 579. Madrid: Imprenta de la viuda de D. Joaquín Ibarra. http://www.sedhc.es/biblioteca/tratado.php?ID_pubD=20.

(17) Fornés, M. (1841). Observaciones sobre la práctica del arte de edificar, p. 49. Valencia: Imprenta de Cabrerizo. http:// www.sedhc.es/biblioteca/tratado.php?ID_pubD=17.

(18) San Nicolás, F.L. (1639). Arte y uso de Architectura, p. 90. Madrid. http://www.sedhc.es/biblioteca/tratado.php?ID_ $\mathrm{pubD}=27$. 
(19) Gómez-Ferrer, M. (1998). Arquitectura en la Valencia del siglo XVI. El Hospital General y sus artífices, p. 144. Valencia: Albatros.

(20) Soler, R. (1986). Estudios previos. Iglesia Sant Tomás i Sant Felip Neri. (Memoria inédita). Valencia: Generalitat Valenciana.

(21) Castellet, L., Soler, R. (2006). Obras de restauración Iglesia de San Bartolomé de la Todolella. Castellón. (Memoria inédita). Valencia: Generalitat Valenciana.

(22) Soler, R., Llopis, V., Soler, A. (2002). Projecte de conservació capella del Santissim Crist. Església Sant Roc d’Oliva. (Memoria inédita). Valencia: Generalitat Valenciana.

(23) Calatayud, S., Soler, R. (2009). Proyecto de Obras de emergencia Iglesia de la Asunción de Carcaixent. (Memoria inédita). Valencia: Generalitat Valenciana.

(24) Soler, R., Soler, A. (2005). Noticias de cúpulas de bóvedas tabicadas: el caso del cimborrio a punta de diamante del Ermitorio de san Marcos, en Olocau del Rey. En Actas I Jornadas de Investigación en Construcción, (pp. 129-148). Madrid: AMIET.

(25) Soler, R., Lizandra, J., Soler, A. (2005). Projecte i Direcció Obra d’emergencia de la cúpula de la Arciprestal de Vilareal, Castelló. (Memoria inédita). Valencia: Generalitat Valenciana.

(26) Heyman, J. (2011). Cáscaras delgadas de fábrica. En Zaragoza, A., Soler, R., Marín, R. (Eds.), Construyendo bóvedas tabicadas, (pp. 295-306). Valencia: Universidad Politécnica.

(27) Soler, R., Martínez, A., Alonso, A. (2006). The eighteenth century brickwork domes in Valencia. En Dunkeld, M., Campbell, J., Louw, H., Tutton, M., Addis, B., Thorne, R. (Eds.), Proceedings of The second Internacional Congress on Construction History, Vol. 3 (pp. 2945-2963). Cambridge: Construction History Society. 